\title{
Über Hüftgelenkersatz, Diagnosen, Gesundheitsmarkt und Demenz
}

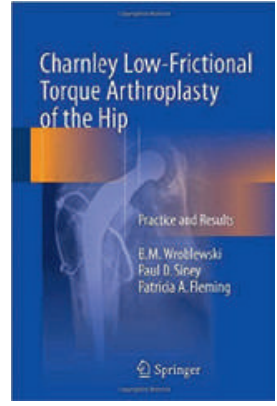

B.M. Wroblewski, Paul D. Siney,

Patricia A. Fleming

Charnley Low-Frictional Torque

Arthroplasty of the Hip

Practice and Results

Springer International Publishing

Switzerland; 2016.

357 Seiten. 159 USD.

ISBN 978-3-319-21319-4

Das soeben von Prof. Wroblewski und Mitarbeitern publizierte Buch über die klinischen Erfahrungen mit der low-frictional torque arthroplasty von Sir John Charnley ist ein Musterbeispiel von seriöser wissenschaftlicher Arbeit. Die seit 1962 sorgfältig lückenlos durchgeführten Kontrollen der mit dem von Charnley entwickelten Hüftgelenkersatz im Wrightington Hospital operierten Patienten zeigen hervorragende Langzeitergebnisse.

Dank der auf der Erfahrungsbasis während mehr als 50 Jahren entwickelten Verbesserungen am Design der Prothese und der Operationstechnik ohne Änderung der Prinzipien Charnleys kann ein Patient mit dem Hüftgelenkersatz nach Charnley mit über 90\% Wahrscheinlichkeit damit rechnen, dass das Gelenk während 30-40 oder sogar mehr Jahren perfekt funktioniert und frei beweglich und stabil bleibt.

Damit ist der Hüftgelenkersatz nach Charnley einzigartig erfolgreich.Es ist zu hoffen, dass der Erfolg weltweit zur Kenntnis genommen wird. Es liegt im Interesse der Patienten, wenn möglichst viele orthopädische Chirurgen zur Durchführung der low-frictional torque arthroplasty ausgebildet werden.

Die Prinzipien des Charnley-Hüftgelenkersatzes lauten wie folgt: Trochanterosteotomie zur idealen Freilegung des Hüftgelenkes und zur Möglichkeit, die Gelenksbelastung durch Änderung der Hebelwirkung zu vermindern,
Prothesenkopf mit 22,225 mm Durchmesser zur Reduktion der Torsion, Verwendung von UHMWPE für die Pfanne, maximale Asepsis und Verwendung von Knochenzement.

Prof. Dr. med. Max Geiser

Gossetstrasse 47, CH-3084 Wabern

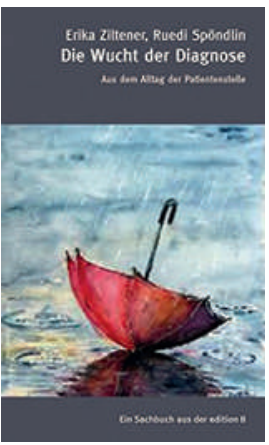

Erika Ziltener, Ruedi Spöndlin Die Wucht der Diagnose

Aus dem Alltag der Patientenstelle Zürich: edition 8; 2015.

176 Seiten. 24 CHF.

ISBN 978-3-85990-263-3

1979 wurde in Zürich die erste Patientenstelle gegründet. Neuere Themen sind Spitalinfektionen oder die Klassiker, wie ungenügende Aufklärung über Behandlungen und Risiken. Zahlreiche Fallbeispiele illustrieren heikle Themen wie Forschung, Impfobligatorium, Betreuung als Pflegefall, Organtransplantationen oder die Tätigkeit der KESB. Das Buch möchte den betroffenen Personen Orientierungshilfe und Unterstützung sein, dem Gesundheitspersonal Denkanregungen bieten und eigenen Handlungsbedarf anzeigen. Politiker sind dort gefordert, wo durch die Ökonomisierung des Gesundheitswesens das Wesentliche verloren geht: dem Menschen helfen, gesund zu werden, Schmerzen zu lindern und ihn im Unglück zu begleiten. Das Buch zeugt von der enormen Erfahrung der Autorin und des Autors. Sie verstehen es, komplizierte Zusammenhänge ohne Abstriche verständlich und sogar spannend darzustellen. Sie überzeugen als neutrale Beobachter und Ratgeber, die ohne Vorurteile kompli- zierte medizinische, juristische und politische Dimensionen transparent und exemplarisch aufdecken. Im Anhang werden die Angebote und Dienstleistungen der Patientenstelle Zürich aufgelistet. Ein Stichwortverzeichnis macht das Büchlein zu einem Nachschlagewerk, das Patienten, Versicherten und medizinischen Fachpersonen gleichermassen zu empfehlen ist.

Dr. med. Erhard Taverna

erhard.taverna[at]saez.ch

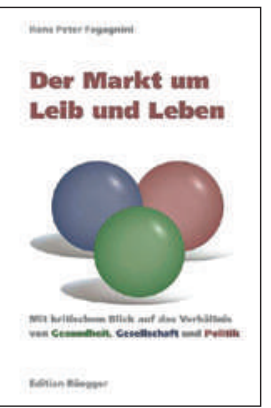

Hans Peter Fagagnini

Der Markt um Leib und Leben

Glarus: Somedia Buchverlag,

Edition Rüegger; 2015.

212 Seiten. 42.90 CHF.

ISBN 978-3-725-31027-2

Einen ungewöhnlichen Schritt wagt Hans Peter Fagagnini mit seinem neuesten Buch. Es trägt denn auch einen besonderen Titel und soll auf nicht ganz alltägliche Weise Einsichten und Beobachtungen zum Besten geben. Der Autor verschafft dem Leser Einblicke darüber, wie wir uns im Geschäft ums Wohlbefinden bewegen, und zeigt mit übersichtlichen Tabellen illustrativ auf, wie die Gesundheit v.a. von Lebensbedingungen abhängt. Zudem führt er den Leser der Frage nach, worin die politische Aufgabe im Gesundheitsmarkt besteht, nämlich im Nachbessern oder Vordenken. Mit überraschenden Gedanken regt er den Leser an, Gesundheits- und Krankheitsaspekte mit unterschiedlichen Perspektiven zu fokussieren.

Selbst, wie er schreibt, mehrfach erfahrener Kunde der Gesundheitsindustrie und frei von 
Interessen, gelingt ihm ein sehr zur Nachahmung empfohlener Weg durch die Landschaft der ganzen Szenerie. Diese hat es bekanntlich in sich, beanspruchen doch ganz unterschiedliche Disziplinen ein Machtwort, wenn es um Bewertung und Zukunft der Gesundheit geht. Philosophische ebenso wie ethische oder anthropologische Stellungsbezüge zeigen unterschiedlichste Zusammenhänge auf, und sie stehen teils quer, teils gleichlaufend mit so profanen Vorstellungen der Betriebswirtschaftslehre oder der Ökonomie. Alledem muss sich die Medizin stellen, nicht um einfach auszuführen, aber auch nicht, um sich in die Abwehr zu begeben.

Wie löst der Verfasser diese Probleme der mehrfachen Kontroversen, die heutzutage rasch um das liebe Geld sich drehen? Indem er nahe beim Menschen bleibt, den er in verschiedenen Rollen sieht: als Patient, als im Beruf stehend oder bereits als Pensionär, als Stimmbürger, als Steuerzahler, aber auch als Träger von Werten. So kommt er stets auf Menschenbilder zu sprechen; sie sind ihm Orientierungsmarken, wenn es um wichtige Entscheide geht. Wonach denn sonst soll man sich orientieren?

Nicht weniger wichtig sind ihm Erfahrungen von früher und die Einschätzung dessen, was die Zukunft bringen könnte. Ist sie breit und hell, oder nur noch alt, oder zieht gar neue Unbill auf? Nach welchem Fixstern richten sich die meisten Menschen aus? Wohl nach der Lebenserwartung. Welche Konsequenzen ergeben sich aus dem Kampf nach immer mehr Lebensjahren? Wird die Medizin diesbezüglich zum Opfer ihrer eigenen Erfolge? Den Dingen so nachgefragt, kommt es vielerorts bekanntlich zu offener Kritik und der Befürchtung, ein Kollaps stehe unmittelbar bevor. Nicht so beim Verfasser, er ist rationaloptimistisch. Was gibt der ganze Gesundheitssektor her? Vieles: eine hohe Wertschöpfung, interessante Berufe, Dienstbereitschaft, Berufung, sehr gute Arbeitsplätze. Und schliesslich ist eine grosse Zahlungsbereitschaft festzustellen. Alles paletti? Das auch wieder nicht. Deshalb ist es dem Verfasser ein Anliegen, das Politikfeld Gesundheit nach allen Regeln der Politik-Kunst auszugestalten, ihm eine Struktur zu vermitteln.

Fazit: Dies ist ein Buch, das zieht, und zwar mehrfach: als Standortbestimmung, als An- leitung zur Festlegung der eigenen Strategie, als Orientierungshilfe, als Lehrmittel der Gesundheitswissenschaft, für Betroffene und deren Angehörige. Als Basis, um in schwierigen Lagen eine Güterabwägung vorzunehmen zu können.

lic. iur. Barbara Züst

barbara.zuest[at]spo.ch

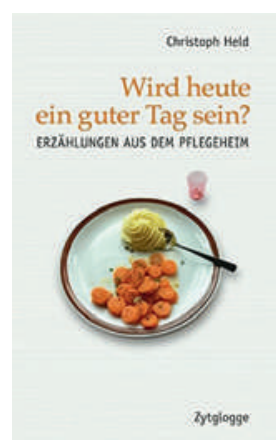

Christoph Held

Wird heute ein guter Tag sein?

Erzählungen

Basel: Zytglogge Verlag; 2010.

128 Seiten. 32 CHF.

ISBN 978-3-729-60800-9

In seinem neuen Buch Wird heute ein guter Tag sein? erzählt uns Christoph Held, Gerontopsychiater und langjähriger Heimarzt der Stadt Zürich, aus der Perspektive des beobachtenden Heimarztes sieben wundervolle Kurzgeschichten aus dem Heimalltag einer Demenzabteilung. Es sind Geschichten über Schicksale von Demenzpatienten, ihren Angehörigen, von Pflegefachpersonen, oder aber auch über eine Heimköchin, welche zur grossen Überraschung der gastronomischen Szene aus einem berühmten Restaurant ins Pflegeheim gewechselt hat. Oder zum Beispiel die Geschichte des Ex-Professors, der im Speisesaal eine Vorlesung hält. Infolge seiner Demenz fällt seine Realität immer wieder in die Zeit von 1968 zurück und der damalige Konflikt mit seiner Tochter, einer 68er-Aktivistin, die ihn damals öffentlich im Audito- rium als «Papiertiger» beschimpft hat, bricht jeweils von neuem aus. In seinen glasklaren Momenten werden ihm jeweils gnadenlos seine Defizite vor Augen geführt. Bis zum Schluss aber bleibt der Professor neugierig über seine Krankheit, die Tochter liest ihm daher trotz fortgeschrittener Krankheit aus medizinischen Fachbüchern zum Thema Demenz vor. Oder da sind die verschiedenen Geschichten des multikulturellen Pflegepersonals, die Originale, die Naturtalente. Christoph Held erzählt von den Spannungen und Schwierigkeiten der kosovarischen und serbischen Hilfspfleger, welche harmonisch zusammen im Pflegeteam arbeiten sollten, während in ihrer Heimat der Kosovo-Krieg tobt. Oder von der sympathischen, überengagierten Pflegerin, welche all ihre Kräfte und Energie für eine fürsorgliche palliative Versorgung der sterbenden Patienten im Pflegeheim einsetzt und sich gegen die Einführung des assistierten Suizids in den städtischen Pflegeheimen sträubt und schwertut.

Wird heute ein guter Tag sein? ist nicht ein Fachbuch im engeren Sinne. Die Sprache ist so gewählt, dass es für ein Laien-Publikum verständlich zu lesen ist, wobei das Buch aber auch für Ärzte und andere Mitarbeiter des Gesundheitswesens äusserst spannend, kurzweilig und empfehlenswert ist. Christoph Held zeigt uns, dass trotz fortgeschrittener Demenzerkrankung die Menschenwürde der Patienten bis zuletzt erhalten bleibt. Ebenfalls zeigt das Buch auf, wie wichtig, spannend, abwechslungsreich und anspruchsvoll die Arbeit auf einer Demenzabteilung ist. Die verschiedenen Geschichten regen uns immer wieder zum Denken an. Der Autor erzählt in einer klaren, schonungslosen, aber sehr empathischen, poetischen Sprache die wunderbaren, berührenden, lieblichen, menschlichen, teilweise brutalen, situationsgebunden humorvollen Geschichten aus dem Heimalltag. Auch als Geschenk eignet sich das Buch hervorragend.

Dr. med. Markus Minder

markus.minder[at]spitalaffoltern.ch 